\title{
Electrically Active Defects in Polycrystalline and Single Crystal Metal Halide Perovskite
}

\author{
Mara Bruzzi ${ }^{1,2,3, *}$, Naomi Falsini ${ }^{1}$ (D), Nicola Calisi ${ }^{4}(\mathbb{D})$ and Anna Vinattieri ${ }^{1,2,3}$ (D) \\ 1 Dipartimento di Fisica ed Astronomia, Università degli Studi di Firenze, Via G. Sansone 1, \\ 50019 Sesto Fiorentino, Italy; naomi.falsini@unifi.it (N.F.); anna.vinattieri@unifi.it (A.V.) \\ 2 Istituto Nazionale di Fisica Nucleare, Sezione di Firenze, Via G. Sansone 1, 50019 Sesto Fiorentino, \\ Firenze, Italy \\ 3 Consorzio Interuniversitario Nazionale per la Scienza e Tecnologia dei Materiali, Sezione di Firenze, \\ Via G. Giusti 9, 50121 Firenze, Italy \\ 4 Dipartimento di Ingegneria Industriale, Università di Firenze, Via S. Marta 1, 50139 Firenze, Italy; \\ nicola.calisi@unifi.it \\ * Correspondence: mara.bruzzi@unifi.it
}

Received: 27 February 2020; Accepted: 27 March 2020; Published: 2 April 2020

\begin{abstract}
We studied electrically active defects in $\mathrm{CsPbBr}_{3}$ polycrystalline films and single crystals samples using the thermally stimulated currents (TSC) technique in the temperature range 100-400 K. Below room temperature, both polycrystalline and single-crystals TSC emission is composed by a quasi-continuum of energy levels in the range $0.1-0.3 \mathrm{eV}$, and capture cross sections $\sim 10^{-21} \mathrm{~cm}^{2}$. Above room temperature, TSC analysis reveals the presence of defect states in the range $0.40-0.52 \mathrm{eV}$ only in polycrystalline samples, whereas these intermediate energy states are absent in TSC detected in single crystals. In polycrystalline films, the occupancy changes of an energy level at $0.45 \mathrm{eV}$ strongly influences the room temperature photoconductivity, giving rise to slow transients due to defect passivation. In single-crystals, where intermediate energy states are absent, the photoconductivity response during illumination is almost stable and characterized by fast rise/decay times, a promising result for future applications of this material in photodetection and dosimetry.
\end{abstract}

Keywords: metal halide perovskite; photoconductivity; defects; thermally stimulated currents

\section{Introduction}

Lead halide perovskite $\mathrm{CsPBBr}_{3}$ is an emerging semiconductor material characterized by extremely promising optical and transport properties for next-generation optoelectronic devices: besides solar cells [1] and light-emitting diodes [2], metal halide perovskites have been taken recently under consideration as photodetectors [3] and dosimeters for medical applications [4]. Nonetheless, their potential application is limited by the poor knowledge about defects and their influence on carrier trapping and recombination effects, detrimental for the device performances [5]. Recently, a work on trap states in perovskite solar cells has been carried out with deep level transient spectroscopy (DLTS) investigation [6]. The DLTS method [7], monitoring changes in the capacitance, can be used only when either a Schottky barrier or a p-n junction is present, so the study has been carried out on a perovskite solar cell. In this way, the investigation is not directly focused on the perovskite material, being the solar cell a device of rather a complex composition of several elements. Instead, a different technique, the thermally stimulated current (TSC) analysis [7], is more effective to investigate the presence of electrically active defects in the bulk material. Indeed, the TSC technique is a powerful tool in this respect, as it can be applied to any electric field profile settled within the sample, including the ohmic configuration. Only very few works on the thermally stimulated current technique applied to $\mathrm{CsPbBr}$ 
are available up to now in literature. A study on single crystal $\mathrm{CsPbBr}$ samples has been recently published, mainly focussing on the low-temperature range below $300 \mathrm{~K}$ [8]. In a more recent report, the TSC analysis has been applied to $\mathrm{CsPbBr}_{3}$ polycrystalline films [9] and the temperature range has been extended to $400 \mathrm{~K}$, to study a wider energy range, including also defects which are active in the temperature range of interest for solar cells and photodetector applications.

Defects are limiting the efficiency of solar cells, photodetectors, and dosimeters, mainly in two ways: acting as nonradiative recombination centers and giving rise to unwanted instability in electrical parameters as e.g., photoconductivity. In solar cells, it is known that nonradiative recombination has an impact on the fill factor $(\mathrm{FF})$, open-circuit voltage $\left(\mathrm{V}_{\mathrm{OC}}\right)[10,11]$ and their hysteretic behavior [12]. In detectors and dosimeters, the nonradiative recombination is responsible for the incomplete collection of the carriers generated by the incident particles/radiation. Moreover, electrically active defects bring instability of the current measured when the device is exposed to a constant radiation flux, due to trapping/detrapping of charged carriers. Signal instability is especially detrimental when the semiconductor film is used as a photodetector/dosimeter. In a photodetector, the sensitivity is given by the current per unit flux of radiation and unit volume, equivalently in a dosimeter, the sensitivity is given by the collected charge at electrodes per unit dose and unit volume. Thus, the current/charge signals must be constant when the radiation flux/dose is constant and their dependence on the radiation flux/dose must be linear; the rise/decay times when switching the beam on and off must be negligible as compared with the operating times typical of the specific application.

This paper is focused on the study of defects responsible for trapping/detrapping effects giving rise to instability in room temperature photoconductivity. We have studied polycrystalline and single crystals $\mathrm{CsPbBr}_{3}$ samples with TSC to determine the energy and capture cross-section of traps in a range of temperatures of interest for photodetector and dosimetry applications. The photoconductivity response has been studied as a function of time to elucidate the role of trapping/detrapping of the main defects revealed by TSC.

\section{Materials and Methods}

Two sets of $\mathrm{CsPbBr}_{3}$ samples have been manufactured and investigated: polycrystalline films and single crystals. The polycrystalline films have been deposited by drop-cast directly on alumina printed circuit board (PCB) specially designed for electrical tests of thin semiconductor films in planar electrodes configuration. PCBs have two/four parallel Au contacts, $20 \mu \mathrm{m}$ thick, a few mm long and $0.4-0.8 \mathrm{~mm}$ spaced. They also hold integrated $\mathrm{Pt}$ temperature sensors and a heater on the backside. We deposited the film starting from a saturated solution of $\mathrm{CsBr}$ and $\mathrm{PbBr}_{2}$, mole ratio 1:1 in dimethyl sulfoxide (DMSO). To remove the solvent, we performed thermal annealing at $150{ }^{\circ} \mathrm{C}$, to produce a dense and continuous layer composed of interconnected $\mathrm{Cs} \mathrm{PbBr}_{3}$ crystallites of the order of a few $\mu \mathrm{m}$ [13]. The annealing temperature is a few degrees under the DMSO boiling point to prevent bubble formation which could cause the destruction of the film. Precursors ratio 1:1 was selected in view to minimizing the impurities in the perovskite material. The XRD/XPS analysis evidenced the good quality of the film with no residual solvents and contaminants. The film was then covered with Poly(methyl methacrylate) (PMMA) to prevent deterioration due to air and water vapor [14]. A PMMA solution was obtained by dissolving the polymer in dichloromethane. The obtained solution was then drop-casted on the sample and, after the evaporation of the solvent, it was observed the formation of a uniform film. The solvent was evaporated at room temperature and in a fume hood. We grew single crystals using a solvent/anti-solvent procedure. In this case, a saturated solution of the $\mathrm{CsBr}$ and $\mathrm{PbBr}_{2}$ 1:1 molar ratio in DMSO has also been obtained. The perovskite solution has been placed in a glass crystallizer and covered with a paper filter. We placed a glass Petri dish above the filter. We inserted the system into a second glass container with hermetic closure. In the second container, we added an antisolvent (in this specific case, methanol), then we closed the system. The antisolvent slowly penetrated the innermost container containing the perovskite solution through the paper, mixed with the solvent and slowly precipitated the perovskite. The slow precipitation allowed for the formation 
of relatively large and orderly crystals [15]. Polycrystalline films and single-crystal samples with dimensions in the order of a few mm mounted on a printed circuit board are shown respectively in Figure 1a,b. Single crystals have been glued on the Au contacts using silver paint and then covered with the PMMA film with the same procedure used for polycrystalline films.

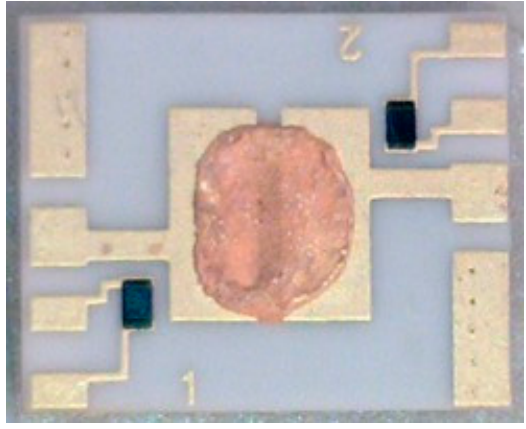

(a)

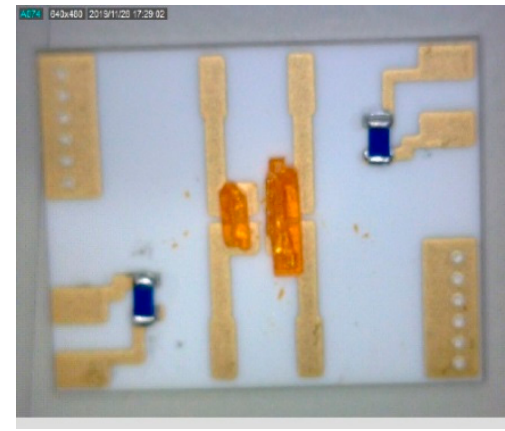

(b)

Figure 1. Photograph of (a) polycrystalline and (b) single crystals $\mathrm{CsPbBr}_{3}$ deposited on alumina $\mathrm{PCB}$ equipped with Au contacts and Pt100 temperature sensors.

Both polycrystalline and single-crystal samples have been fully characterized by photoluminescence (PL) spectroscopy in a temperature range from $10 \mathrm{~K}$ to $300 \mathrm{~K}$. PL measurements were performed in a quasi-backscattering geometry exciting the samples by a continuous wave $(\mathrm{CW})$ laser diode operating at $405 \mathrm{~nm}$ with an excitation intensity of about $10 \mathrm{Wcm}^{-2}$ (an illuminated spot of about $100 \mu \mathrm{m}$ diameter). Drop-cast and single-crystal PL spectra at $10 \mathrm{~K}$, shown in Figure 2a,b, respectively, are similar to recent literature (see [16] and references therein). Figures also show the joint density of states (DOS) extracted from the PL spectra, according to [16]. In Figure 2b, BE and FE indicates the bound and free exciton emission, respectively. The high quality of the single-crystal samples is proven by the evidence of the free exciton emission (FE) with a negligible Stokes shift respect to the DOS resonance at $2.32 \mathrm{eV}$. The inhomogeneous broadening of the PL of the drop-cast sample turns out to be $25 \mathrm{meV}$, in agreement with values in the literature (see for instance [17]).

Figure $2 \mathrm{c}$ shows the normalized spectrally integrated intensity of the PL as a function of the inverse of the temperature for $\mathrm{CsPBr}_{3}$ single-crystal and drop-cast samples (red and blue dots, respectively). In the figure, the corresponding fitting curves are also shown for the two samples (orange and light blue curves, respectively). The fitting function of the experimental data is an Arrhenius curve with two activation energies: the first activation energy is $13 \pm 5 \mathrm{meV}$ and the second one is $52 \pm 9 \mathrm{meV}$ for both samples within the experimental error. Those values are in good agreement with the literature data where a significant spread of the activation energies are found dependant if one or two non-radiative channels are considered [18,19].

We characterized the current-voltage (IV) characteristics of our samples as follows. First, we applied a voltage across the electrodes. We then observed a transient of the current with time as described in [20]. This effect is probably due to ion migration due to the applied electric field [20,21]. The current stabilizes after a few minutes, reaching an equilibrium state. This stabilization process is carried out also before the filling procedures and the TSC analyses. Every measurement is started after waiting a few minutes after having applied bias for current stabilization. IV in the dark are measured when stationary conditions are achieved at each voltage step. We used voltages in the range of $\pm 5 \mathrm{~V}$, to keep a sufficiently low electric field within the material. In fact, a very recent study on $\mathrm{CsPbBr}_{3}$ [21] shows that if a very high electric field is applied in single crystals, defect proliferation phenomena originating from ion migration occur. To evidence this effect, in [21], up to $100 \mathrm{~V}$ was applied to single crystals of approximately the same size as ours. Our IV curve shows a linear response, indicating an ohmic regime, without significant hysteretic behavior, which would indicate the effect of defect proliferation 
due to ion migration. Resistivity at room temperature is about $10^{9} \Omega \mathrm{cm}$ and $2 \times 10^{8} \Omega \mathrm{cm}$ respectively for polycrystalline and single-crystal samples.

Previous measurements on similar samples [9] showed a p-type conductivity with mobilities of about $10 \mathrm{~cm}^{2} / \mathrm{Vs}$ at room temperature. In the following, we will assume that in the temperature range investigated in this study, mobility is dominated by phonon scattering, namely, it follows a dependence $\mu(T) \propto T^{-3 / 2}$ during the TSC scans [22]. This is in agreement with what was observed in the past with hybrid halide perovskites [23,24].

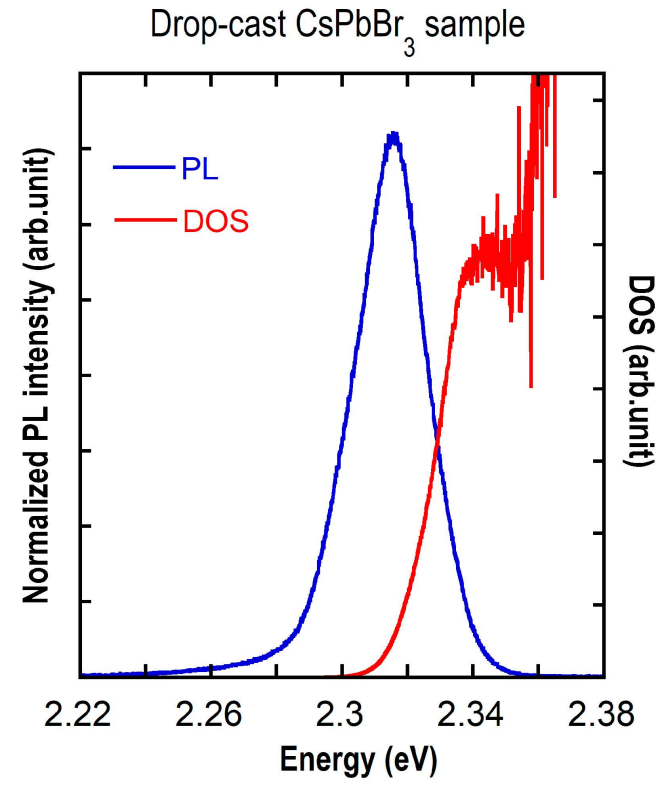

(a)

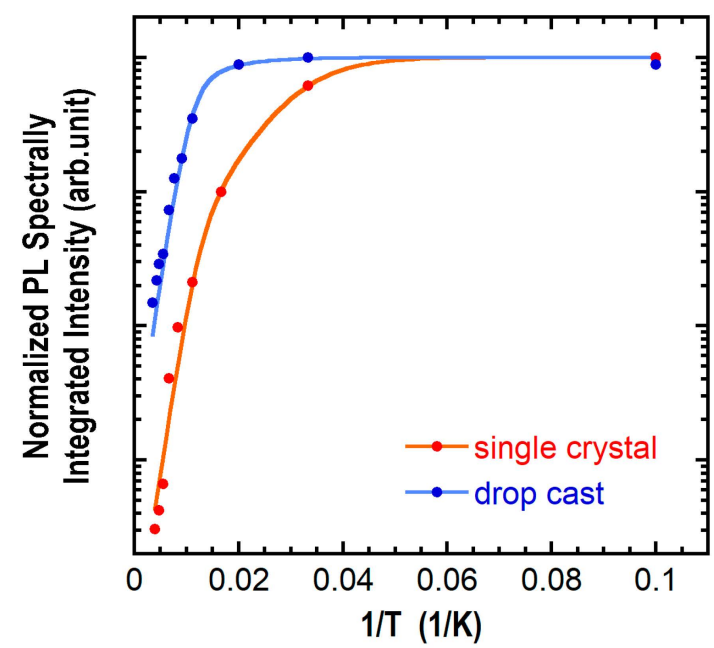

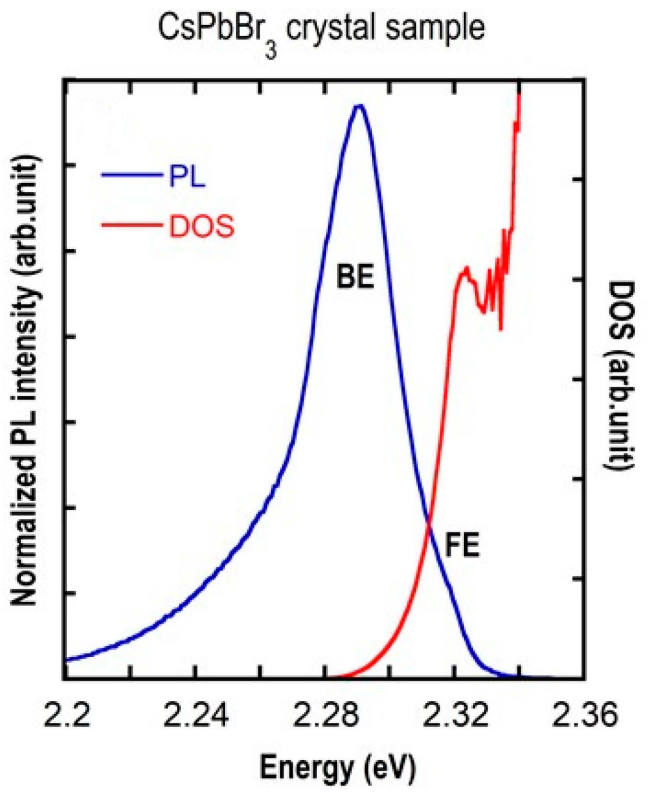

(b)

(c)

Figure 2. PL spectra of $\mathrm{CsPbBr}_{3}$ (a) drop-cast and (b) single-crystal samples at $10 \mathrm{~K}$. The red curves are the joint density of states (DOS) as extracted from the PL spectra according to [16]. BE and FE indicate the bound and free exciton emission, respectively; (c) Normalized spectrally integrated intensity of the $\mathrm{PL}$ ( $\log$ scale) as a function of the inverse of the temperature is shown for $\mathrm{CsPbr}_{3}$ single-crystal and drop-cast samples (red and blue dots, respectively) along with the corresponding fitting curves (orange and light blue curves, respectively). 
We measured the thermally stimulated current (TSC) in the temperature range 100-400 K, using a Keithley 6517 electrometer to read-out the current and to bias the sample under test. We performed the temperature scans by inserting the sample-holder within liquid nitrogen vapors inside a dewar to ensure stable temperatures, minimize thermal inertia, and reduce the possible mismatch between the sample and the thermometer. We controlled the temperature during scans by utilizing Pt100 sensors controlled by a Lakeshore DRC91C. A TTi QL564P power supply was used for the $20 \Omega$ resistor-heater placed underneath the sample, to heat-up the samples during the thermal scans. Samples were illuminated by a blue $(400 \mathrm{~nm}, 0.8 \mathrm{~mW})$ LED placed in front of it inside the cryostat during priming.

\section{Results}

\section{Thermally Stimulated Currents}

First, we observe that TSC is not able to select between hole- and electron-traps. We will, therefore, refer in the following to a majority trap occurring in the p-type material. Current flowing between two ohmic contacts is given by:

$$
I=q p v_{d} A
$$

With $p$ free holes concentration, $q$ electron charge absolute value, $A$ surface normal to the electric field $\mathrm{F}, v_{d}=\mu_{p} F$, drift velocity $\left(F=V_{b} / d, V_{b}\right.$ potential drop at electrodes, $\mathrm{d}$ distance between electrodes), $\mu_{p}$ hole mobility. In this model, we consider the presence of at least one active trap and one recombination center. The rate of traps filled with holes, $p_{t}$, change with time following the expression [7]:

$$
\frac{d p_{t}}{d t}=c_{p}\left(N_{t}-p_{t}\right)-e_{p} p_{t}
$$

with $N_{t}$ total trap concentration. $c_{p}$ and $e_{p}$ capture and emission coefficients, respectively. They depend on trap parameters $E_{t}$ - energy level (distance from valence band edge if hole-trap) and $\sigma$-capture cross-section as:

$$
c_{p}=\sigma v_{t h} p ; e_{p}=N_{v} v_{t h} \sigma e^{\frac{E_{v}-E_{t}}{K T}}
$$

with $N_{v}$ effective density of states at valence band edge, $v_{t h}$ hole thermal velocity. In stationary conditions we get:

$$
\frac{d p_{t}}{d t}=0 ; T O=\frac{p_{t}}{N_{t}}=\frac{c_{p}}{c_{p}+e_{p}}
$$

The trap occupancy is fixed by the ratio between $c_{p}$ and $c_{p}+e_{p}$. We note it is independent of the cross-section $\sigma$ :

$$
T O=\frac{p}{p+N_{v} e^{\frac{E_{v}-E_{t}}{K T}}}
$$

Before undergoing a TSC measurement, it is necessary to fill the traps with carriers, a process called priming. The priming process is carried out either by exposing the sample to light or by delivering an electrical pulse to the sample. Usually, this is performed by illuminating at a fixed temperature, $T_{i}$, the sample with photons with energy above the bandgap. Charged carriers promoted in the conduction/valence bands will be easily trapped into empty energy levels and remain frozen until heating thermally excites them again towards the respective conduction/valence bands. The increase of trap occupancy TO due to this filling process is carried out at a low temperature to leave the charge carriers frozen within the traps afterward. When the sample is heated with a constant heating rate $\beta$, traps begin to release their charge to the corresponding band. If no-retrapping is considered during this heating process, Equation (2) reduces to $\frac{d p_{t}}{d t} \sim-e_{p} p_{t}$ and the concentration of occupied traps decreases following the law:

$$
p_{t}(t)=p_{t}(0) e^{-\int_{0}^{t} e_{p}(t) d t}=p_{t}(0) e^{-\frac{1}{\beta} \int_{T i}^{T} e_{p}(T) d T}
$$


where $p_{t}(0)$ is calculated from the trap occupation before the heating process starts. During heating in the dark, the free holes concentration in the valence band changes due to the emptying of the trap: $\frac{d p}{d t} \approx-\frac{d p_{t}}{d t}$. The excess of carriers recombines at the recombination center as: $\frac{d p}{d t} \approx \frac{p}{\tau_{e f f}}$, having assumed $\tau_{\text {eff }}$ as the effective lifetime before recombination, taken as a constant in this model [7]. So, we get:

$$
p \approx \frac{d p}{d t} \tau_{e f f} \approx-\frac{d p_{t}}{d t} \tau_{e f f} \approx e_{p} p_{t} \tau_{e f f}
$$

The TSC current signal finally results:

$$
I_{T S C}=q \mathrm{e}_{\mathrm{p}} \tau_{e f f} v_{d} A p_{t}\left(T_{i}\right) e^{-\frac{1}{\beta} \int_{T i}^{T} e_{p}(T) d T}=q \mathrm{e}_{\mathrm{p}} d A p_{t}\left(T_{i}\right) e^{-\frac{1}{\beta} \int_{T i}^{T} e_{p}(T) d T}
$$

Keeping as effective time $\tau_{\text {eff }}$, the transit time of the released charges moving across electrodes in the presence of the electric field $F: \tau_{e f f}=\frac{d}{\mu_{p} F}$, and $v_{d}=\mu_{p} F$ the drift velocity. In our experiments, first, we have performed a set of measurements with filling temperature around $290 \mathrm{~K}$ and then heating up to $380 \mathrm{~K}$, to study deeper traps active just above room temperature. Table 1 summarizes the parameters used in our TSC experiments.

Table 1. Parameters used in the TSC experiments.

\begin{tabular}{cc}
\hline Parameters & \\
\hline Temperature rate $[\mathrm{K} / \mathrm{s}]$ & $0.08-1.50$ \\
Effective hole mass & $0.128 \mathrm{~m}_{\mathrm{e}}$ \\
$\mathrm{V}_{\mathrm{b}}$ [Volt] & $2-5$ \\
d electrodes distance $[\mathrm{mm}]$ & $0.4-0.8$ \\
A polycrystalline film $\left[\mathrm{mm}^{2}\right]$ & $2 \times 10^{-2}$ \\
A single crystal $\left[\mathrm{mm}^{2}\right]$ & 2 \\
Capture cross section $\sigma\left[\mathrm{cm}^{2}\right]$ & $10^{-22}-10^{-20}$ \\
\hline
\end{tabular}

Then, we investigated shallower levels by filling at $100 \mathrm{~K}$ and then heating up to room temperature. Figure $3 \mathrm{a}, \mathrm{b}$ show examples of priming processes carried out by illuminating a polycrystalline sample with the blue LED at room temperature. During illumination, the current slowly increases under a constant intensity, indicating the filling of traps. Increasing the exposure duration, the filling process proceeds further and photocurrent keeps rising. After the light is switched off, the current shows a sudden step and a slow decay, due to the discharge of those traps with energy levels that can quickly release the trapped carriers at that temperature. After the light is switched off, the current stabilizes itself to a higher value than the one before illumination, an effect that is clearly visible in Figure $3 \mathrm{~b}$. This phenomenon, called persistent current, could be explained considering the increase in trap occupancy caused by the illumination. In fact, in stationary conditions shown in Equation (5), an increased TO brings an increase in the free hole concentration $p$, due to charge neutrality, and in turn, to a higher current in Equation (1). This new stationary condition is due to the deepest traps that do not discharge at room temperature, they need to be heated to a higher temperature in view to discharge them. The measurement of the TSC current is performed in particular to have evidence of these deeper traps, so we waited a decay time of a few minutes to discharge completely traps active at the initial temperature $T_{i}$. Figure 4 shows the TSC measured after the priming process of Figure $3 a$, together with a TSC measured with the same bias voltage and heating/cooling rates after a long period of storage in ambient light at room temperature. TSC curves in Figure 4 correspond to different pumping of the same broad structure that appears almost saturated after long storage at ambient light. We note that, in Figure 4 , the cooling curves for different $t_{\text {fill }}$ are overlapping, whereas the heating curves are different. 

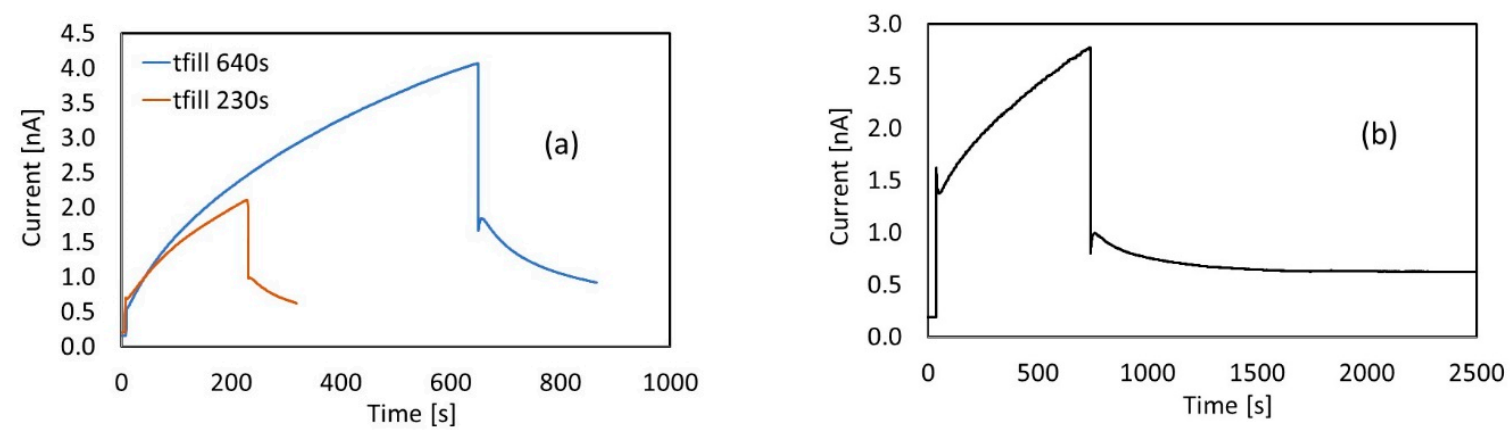

Figure 3. Photoconductivity of polycrystalline $\mathrm{CsPbBr}_{3}\left(400 \mathrm{~nm} \mathrm{LED}, T_{i}=290 \mathrm{~K}, V_{b}=5 \mathrm{~V}\right)$ : (a) two priming with increasing duration; (b) priming process and afterward with light off, to evidence the presence of persistent currents.

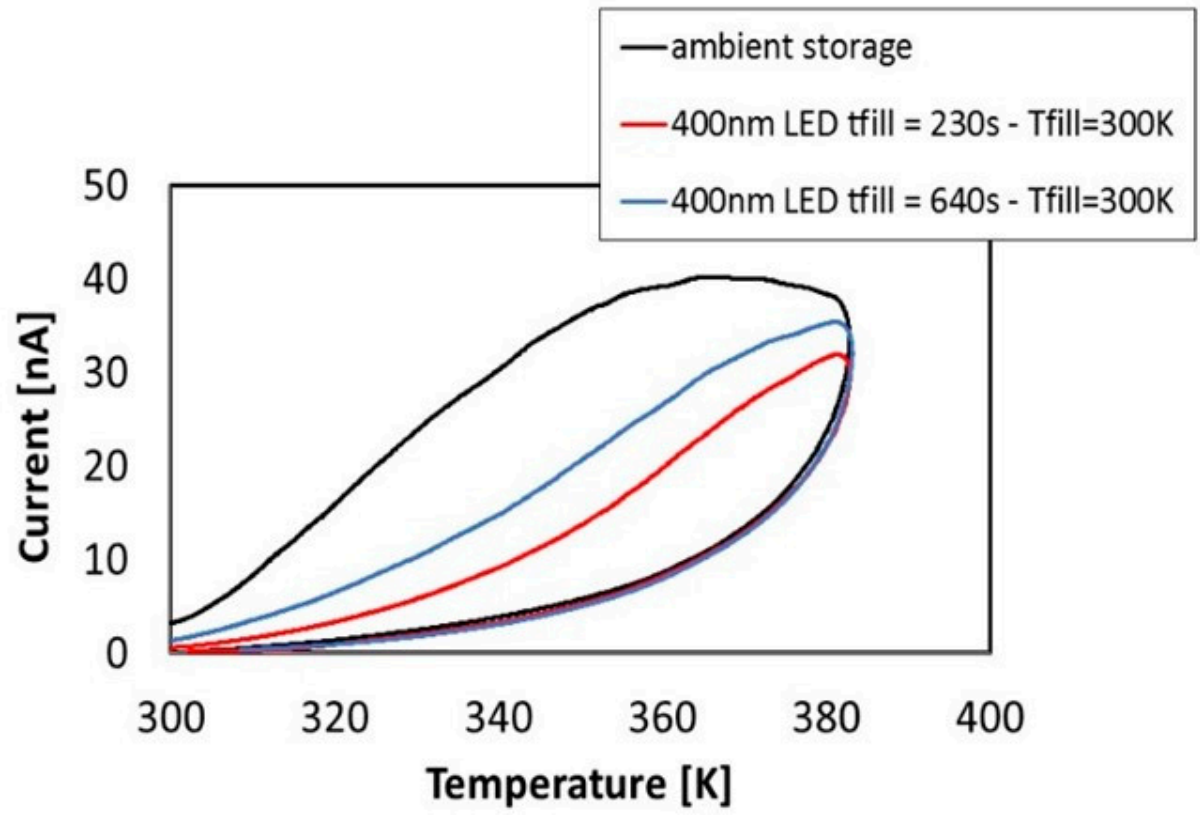

Figure 4. Thermally stimulated currents measured after the two priming processes shown in Figure $3 \mathrm{a}$ as compared with the one measured with the same sample after storage in ambient light, same $T_{i}=300 \mathrm{~K}$, $V_{b}=5 \mathrm{~V}$ and heating and cooling rate $\beta=0.08 \mathrm{~K} / \mathrm{s}$.

To inspect the nature of the energy levels contributing to this broad TSC signal, we have fitted these curves using Equation (8). Figure 5a-c show results: best fits have a dominant component at $T_{\text {peak }}$ $=360 \mathrm{~K}$, accompanied by two minor components: a shoulder at a higher temperature, which accounts also for the background current measured during cooling, Table 2 summarizes energy levels $E_{t}$ of defects best fitting to the measured TSC curves of Figure 4, referring to A: long term ambient storage; B: filling time $230 \mathrm{~s}$; C: filling time $640 \mathrm{~s}$.

Table 2. Energy Levels $E_{t}$ measured of TSC components measured in the range 290-400 K. Trap occupancy TO measured for each energy level depending on the different filling processes at room temperature, A: long term storage; B: filling time $230 \mathrm{~s}$; C: filling time $640 \mathrm{~s}$.

\begin{tabular}{cccc}
\hline $\mathbf{E}_{\mathfrak{t}}[\mathbf{e V}]$ & TO-A [\%] & TO-B [\%] & TO-C [\%] \\
\hline 0.41 & 7.7 & 0.0 & 0.0 \\
0.45 & 22.9 & 5.5 & 12.1 \\
0.52 & 33.5 & 47.1 & 42.6 \\
\hline
\end{tabular}


The three TSC spectra are best-fitted with the same defects, with different trap occupancies due to a different filling duration. Total trap concentrations are of the order of $10^{18} \mathrm{~cm}^{-3}$. Such high defect concentrations have been already observed in lead halide perovskite solar cell structures [8] and similar samples [6]. Trap occupancy of the shallowest defect is either small or negligible: being peaked at $340 \mathrm{~K}$, close to room temperature, it fades away very quickly, so in B and C measurements the long decay time adopted is sufficient to make it disappear. Almost doubling filling time duration, the peak at $0.45 \mathrm{eV}$ trap occupancy becomes almost twice, this effect smoothens towards the saturation value measured for long term ambient storage, about $23 \%$. Increasing filling time duration has no effect on the TSC component peaked at the highest temperature, indicating that its occupancy is only slightly perturbed by light exposure at room temperature. So, we may conclude that the main TSC component responsible for the photoconductivity changes in Figure 3a is due to the energy level at $0.45 \mathrm{eV}$ as was shown by numerical fitting the photoconductivity of similar samples in [6].
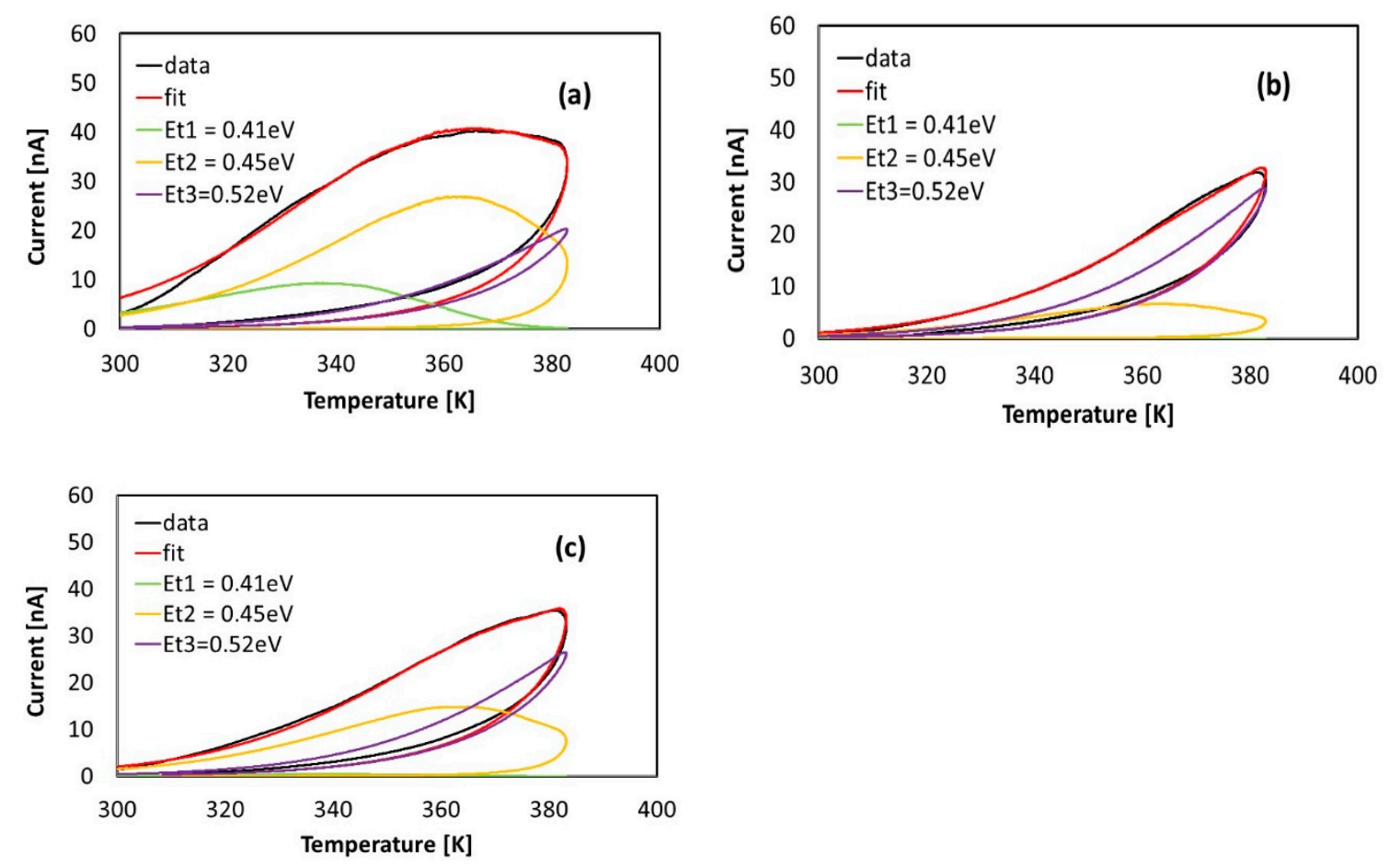

Figure 5. Thermally stimulated currents, TSC peaks calculated using Equation (8) and best fit obtained by the sum of data shown in Figure 3 measured after: (a) ambient storage; (b) and (c) priming with LED $400 \mathrm{~nm} T_{i}=290 \mathrm{~K}$ and $t_{\text {fill }}=230 \mathrm{~s}$ and $640 \mathrm{~s}, V_{b}=5 \mathrm{~V}$ and heating cooling rate $\beta=0.08 \mathrm{~K} / \mathrm{s}$.

Summarizing, heating curves are dominated by the charge released from the energy level at $0.45 \mathrm{eV}$. Changing $\mathrm{t}_{\text {fill }}$, the trap occupancy before heating at $0.45 \mathrm{eV}$ level changes strongly and thus does the heating current. Conversely, the cooling curve is almost completely dominated by the charge released by the trap with energy level at $0.52 \mathrm{eV}$, which trap occupancy is almost independent of $\mathrm{t}_{\text {fill }}$.

Remarkable differences are found when studying single-crystal $\mathrm{CsPbBr}_{3}$ samples. The same priming procedure and TSC analysis have been carried out: Figure $6 \mathrm{a}, \mathrm{b}$ show, respectively, the photoconductive response during priming with the $400 \mathrm{~nm}$ LED at room temperature $V_{b}=5 \mathrm{~V}$, and the following TSC heating/cooling cycle performed at the same bias and temperature rate $\beta=0.08 \mathrm{~K} / \mathrm{s}$. An almost squared photoconductive response during priming is found, with the absence of persistent currents when the light is switched off, and a negligible TSC released during the following thermal cycle in the range of 300-380 K. 

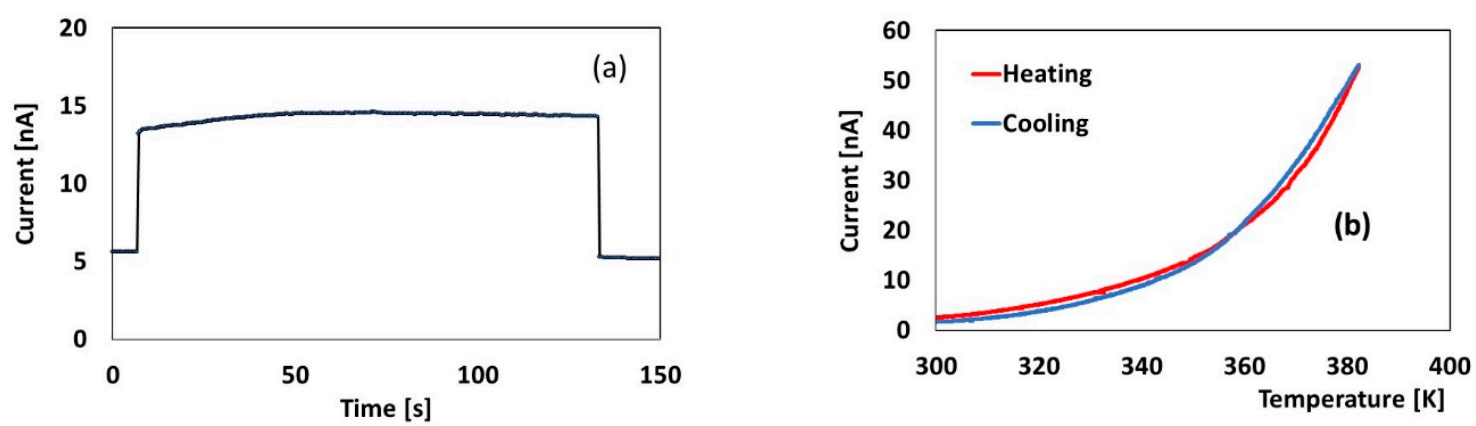

Figure 6. (a) Photoconductivity measured during the priming process on a single crystal $\mathrm{CsPbBr}_{3}$ sample illuminated with a $400 \mathrm{~nm}$ LED at $T_{i}=290 \mathrm{~K}, V_{b}=2 \mathrm{~V}$; (b) TSC cycle (heating and cooling) measurement carried out after priming, showing the absence of thermally stimulated peaks.

The TSC investigation has been carried out at lower temperatures by placing the sample holder in liquid nitrogen vapors inside the dewar. Filling of drop-casted and single-crystal samples has been carried out at $T_{i}=100 \mathrm{~K}$ with the same LED source and then performing a TSC measurement up to $250 \mathrm{~K}$. Figure 7a,b show results for single-crystal and polycrystalline films. The TSC signal has a complex and broadened structure. For polycrystalline samples, results agree with those shown in previous works [9] where a quasi-continuum of states was observed with energy levels in the range of 0.11 to $0.27 \mathrm{eV}$ (not shown in Figure 7). For single crystals, our numerical fit found a set of components in the range of $0.15-0.27 \mathrm{eV}$, almost equally spaced by $25 \mathrm{meV}$.
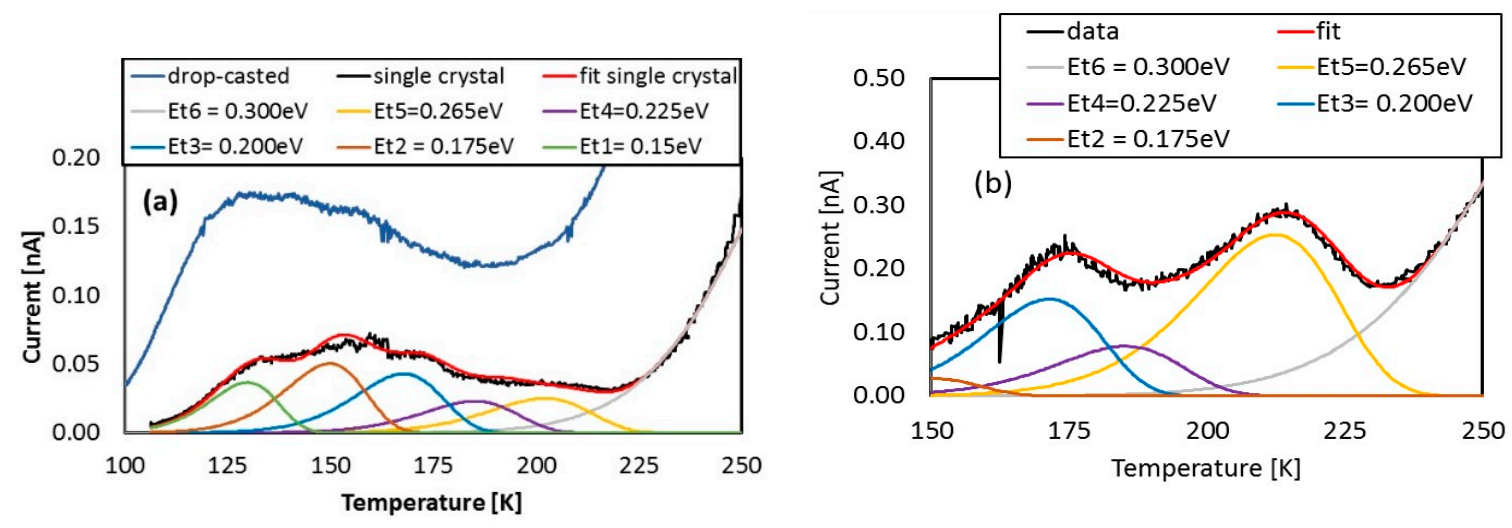

Figure 7. TSC measurement carried out on a single-crystal CsPbBr3 sample illuminated with a $400 \mathrm{~nm}$ LED, $V_{b}=2 \mathrm{~V}$ filling at (a) $T_{i}=100 \mathrm{~K}$ and (b) $T_{i}=150 \mathrm{~K}$. The best fit is carried out considering the same set of thermally stimulated components.

In particular, when filling at $150 \mathrm{~K}$, see Figure $7 \mathrm{~b}$, we found two main components with $\mathrm{T}_{\text {peak }}=175 \mathrm{~K}$ and $210 \mathrm{~K}$, we associate to energy levels at $0.20 \mathrm{eV}$ and $0.26 \mathrm{eV}$, with almost the same capture cross-sections. A shoulder at high temperature is attributed to a defect at $0.30 \mathrm{eV}$.

\section{Discussion}

First, we compare our results on trap parameters measured in $\mathrm{CsPBBr}_{3}$ with those found in the literature. Almost all studies on defects in perovskites have been focussed on methylammonium lead iodide perovskite solar cells. As an example, Duan et al. [25] have identified a defect state at $0.16 \mathrm{eV}$ above the valence band plus a deeper level defect above $0.3 \mathrm{eV}$, defect densities are about $10^{16} \mathrm{~cm}^{-3}$. Baumann et al. [26] performed TSC measurements in methylammonium lead iodide perovskite solar cells assigning a peak to trap states with activation energies around $0.50 \mathrm{eV}$. Laplace's current DLTS showed the presence of two trap levels at $0.17 \mathrm{eV}$ and $0.20 \mathrm{eV}$ from the band edge, capture a cross-section of the order of $3-4 \times 10^{-20} \mathrm{~cm}^{2}$ [27]. In [6], a dominant donor level with an 
energy of $0.2 \mathrm{eV}$ below the conduction band and another two deep traps are found for holes and for electrons with energies of $0.57 \mathrm{eV}$ and $0.74 \mathrm{eV}$, respectively. Here, the capture cross-sections lie in the range of $10^{-15}-10^{-17} \mathrm{~cm}^{2}$. Only very few works on thermally stimulated currents applied to $\mathrm{CsPbBr}_{3}$ are available up to now in the literature. A study on single crystal $\mathrm{CsPbBr}_{3}$ [8] analyzed TSC spectra using SIMultaneous-Peak Analysis (SIMPA) in the assumption that any measured spectral emission could be given by an arbitrary sum of components from a multitude of energy levels acting independently of each other. As a result, single isolated TSC emissions have often been interpreted as the result of the sum of several, not-resolved TSC peaks, with energy levels up to $0.6 \mathrm{eV}$ and capture cross-sections in the range of $10^{-14}-10^{-17} \mathrm{~cm}^{2}$. The range of energy levels found in the literature are consistent with the present study, as well as that of trap concentrations, while capture cross-sections are much higher than in our case. This problem arises probably because often the energy and cross-section values are evaluated together using an Arrhenius plot where only the peak temperature is considered and not the whole TSC spectrum. In fact, the energy level and cross-section are both determining the shape of the entire spectrum and not only its peak temperature. To evidence this, Figure 8 shows how different couples $\left(E_{t}, \sigma\right)$ may originate the same peak temperature but not the same peak shape. Here, the relative TSC signal extracted from data for the 210K TSC component is fitted using Equation (8). This relative TSC signal is obtained by subtracting from the TSC curve shown in Figure $7 \mathrm{~b}$ all the other TSC components but the one attributed to the peak at $210 \mathrm{~K}$. In Figure 8, simulated and experimental current curves are normalized to their highest value at maximum.

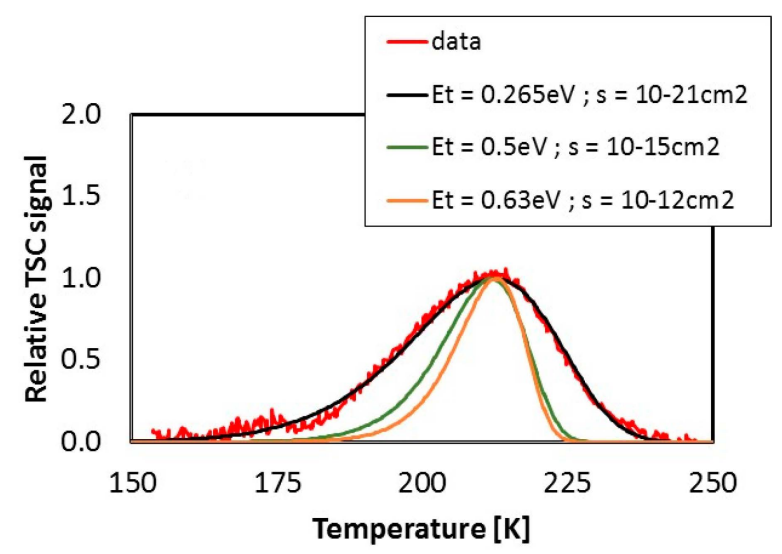

Figure 8. Relative TSC signal extracted from data for a single TSC component is fitted using Equation (8) with different couples $\left(E_{t}, \sigma\right)$. The relative TSC signal is obtained by subtracting from the TSC curve shown in Figure $7 \mathrm{~b}$ all the other TSC components but the one attributed to the peak at $210 \mathrm{~K}$, in view to study a single TSC emission.

If we evaluate the energy of the defect from the position of the peak temperature, in principle, the three couples of values are all fine. Nonetheless, only the couple with $E_{t}=0.265 \mathrm{eV}$ and $\sigma=10^{-21} \mathrm{~cm}^{2}$ fits well the entire TSC signal. In our previous studies, independent measurements of the capture cross-section have been carried out [9] showing that these defects are characterized by very low cross-sections, in the range of $10^{-20}-10^{-22} \mathrm{~cm}^{2}$, as interpreted here and e.g., in [27].

Trap states observed with our TSC analysis have been attributed to lattice defects in $\mathrm{CsPbBr}_{3}$ on the basis of first-principle calculations made in previous works [28]. In this discussion, we divide defects into three classes, mainly depending on their energy with respect to the bandgap value, $2.3 \mathrm{eV}$ : shallow defects, almost fully ionized at room temperature; intermediate states, which contribute to room temperature photoconductivity instability through trapping/detrapping effects; midgap states, behaving mainly as recombination centers.

We observe that, if compared to conventional semiconductors like GaAs, CdSe, InP, considered "defect intolerant" as they are dominated mainly by mid-gap states, $\mathrm{CsPbBr}_{3}$ is potentially "defect tolerant", as it is mostly populated by shallow and intermediate intragap defect states [29]. This can be 
explained considering that, following the hydrogenic model, shallower energy levels are generally due to the combination of small effective masses, $m^{*}$, and high static dielectric constant, $\varepsilon_{r}$ :

$$
E_{n} \propto-\frac{m *}{\varepsilon_{r}^{2}}
$$

So a level, for example, that in silicon is a few tens of meV, reduces to few $m e V$ in $\mathrm{CsPbBr}_{3}\left(m^{*}=\right.$ $0.13 m_{e}$ and $\varepsilon_{r}=29.37$ ) [30-32].

We first consider the shallowest levels in the range $0.1-0.2 \mathrm{eV}$ from the valence/conduction band. In [18], they are associated with vacancy and interstitials, $\mathrm{V}_{\mathrm{Cs}}, \mathrm{V}_{\mathrm{Pb}}$ (acceptors) $\mathrm{V}_{\mathrm{Br}}, \mathrm{Cs}_{\mathrm{i}}$ (donors), as well as to antisite defects such as $\mathrm{CsBr}$, PbCs, (donors) and $\mathrm{BrCs}$ (acceptor). In our TSC study, these defects are present in both polycrystalline films and single crystals. Mainly in the range $0.11-0.27 \mathrm{eV}$, they are characterized by very low capture cross-sections around $10^{-21} \mathrm{~cm}^{2}$. These results are in agreement with those found e.g., for methylammonium lead bromide $\left(\mathrm{MAPbBr}_{3}\right)$ single crystals in which energy levels at $E_{t}=0.11,0.17,0.204 \mathrm{eV}$ were found with capture cross-sections in the range $\sigma=3-5 \times 10^{-20} \mathrm{~cm}^{2}$ [23].

Instability of the photoconductivity in a semiconductor exposed to radiation is usually due to trapping/detrapping defects. The ability to trap and detrap carriers by a defect is ruled out by capture and emission coefficients, which mainly depend on both $E_{t}$ and $\sigma$. Most detrimental defects are those where $E_{t}, \sigma$ give rise to time constants $1 / c_{p}, 1 / e_{n}$ of the same order of magnitude of the collection times typically used when the device is operated. As emission constant is strongly dependent on the temperature $T$, the operation temperature is a crucial parameter when focussing on the photoconductivity measurement. Using $E_{t}, \sigma$ obtained from our TSC analysis for these defects, we find that these traps at room temperature are completely ionized and their contribution to transient photoconductivity effects is negligible.

The second class of levels calculated using ab-initio calculations in $\mathrm{CsPbBr}_{3}$ [28] are intermediate energy levels in the range of $0.30-0.65 \mathrm{eV}$. They are mainly assigned to $\mathrm{Pb}_{\mathrm{i}}$ and $\mathrm{PbBr}$ (donors). In our TSC study, the presence of a current shoulder at $250 \mathrm{~K}$ in Figure $7 \mathrm{a}$,b evidences the effect of a $0.30 \mathrm{eV}$ energy state active below room temperature both in polycrystalline and single crystals. Above room temperature, our TSC measurements show the presence of intermediate levels in the range $0.40-0.50 \mathrm{eV}$ only in polycrystalline films, whereas in single crystals they are not present. They have capture cross-sections of about $10^{-21} \mathrm{~cm}^{2}$ and concentrations up to $10^{18} \mathrm{~cm}^{-3}$. These intermediate states are characterized by $E_{t}, \sigma$ giving rise to time constants for capture and emission of the same order of magnitude of the collection times typically used in room temperature photoconductivity measurements e.g., for dosimetry [4]. In this range, the dominant energy level is $0.45 \mathrm{eV}$ : its trap occupancy is significantly changing depending on the time of exposure to light. Correspondingly, the photoconductivity measured with polycrystalline samples during the filling process at room temperature (see Figure 3a,b) is characterized by slow transients, probably related to the passivation of this defect. This is in agreement with results reported in [9], where the photoconductivity transient measured with similar polycrystalline samples was explained in terms of charge/discharge of an energy level at $0.45 \mathrm{eV}$. The persistent current observed after irradiation in Figure $3 \mathrm{~b}$ could be an effect of the changed trap occupancy of the intermediate energy levels. Conversely, the room temperature photoconductivity measured with single-crystal samples is quite stable, characterized by fast rise/decay times and no persistent currents after the light is switched off. This is in agreement with the fact that no defects in the range of $0.40-0.50 \mathrm{eV}$ are detected in these samples by TSC.

The third class of electrically active defects is that originating midgap levels, mainly responsible for electron-hole recombination effects. In $\mathrm{CsPbBr}_{3}$, ab-initio calculations evidenced the presence of two deep energy levels, at $1.0 \mathrm{eV}$ and $1.4 \mathrm{eV}$, related to two different charged states of the $\mathrm{BrPb}$ antisite defect (acceptors) [29]. In our TSC study, there is no evidence of these defects. The reasons why we do not see them can be explained as follows. First, if they are characterized by capture cross-sections of the same order as the other energy levels, they should be activated, in TSC analysis, at temperatures much higher than our highest limit, $\mathrm{T}=400 \mathrm{~K}$. The second reason is that if they behave as non-radiative 
recombination centers, annihilating free carriers of different charges, they probably do not contribute to the TSC signal. In fact, the presence of recombination centers is generally evidenced indirectly by measuring the minority diffusion length and minority charge lifetime, which is not under study in this work.

In conclusion, as expected, we found a more complex trap distribution in polycrystalline films than in single-crystal samples. This is in agreement with the higher degree of lattice disorder of the polycrystalline network, also proven by PL measurements carried out on the two materials (see Figure 2a,b). In particular, the absence of the intermediate intra-gap states in single-crystals suggests that these levels are associated with defects that in polycrystalline films, are mainly located at boundaries surrounding the micron-sized crystallites. Our results on single-crystal samples, showing the absence of intermediate states related to the instability of the room temperature photoconductivity response, opens the way to potential future applications of this material in photodetection and dosimetry.

Author Contributions: Conceptualization, M.B. and A.V.; methodology, M.B., N.C., A.V.; software, M.B.; validation, M.B., N.F. and A.V.; formal analysis, M.B.; investigation, M.B., N.C., N.F.; resources, M.B. and A.V.; data curation, M.B., N.C., N.F., A.V.; writing-original draft preparation, M.B.; writing-review and editing, M.B., N.C., N.F., A.V.; visualization, M.B., N.C., N.F., A.V.; supervision, M.B. and A.V.; project administration, M.B.; funding acquisition, M.B. All authors have read and agreed to the published version of the manuscript.

Funding: This research received funding from Istituto Nazionale di Fisica Nucleare, Commissione Nazionale 5, PERO2 Project, INFN, Italy.

Acknowledgments: We wish to thank Matteo Intravaia, now at Dipartimento di Ingegneria dell'Informazione e Scienze Matematiche, University of Siena, Italy, for technical assistance in some of the photoconductivity measurements.

Conflicts of Interest: The authors declare no conflict of interest.

\section{References}

1. Sutton, R.J.; Eperon, G.E.; Miranda, L.; Kamino, E.S.; Patel, J.B. Bandgap-Tunable Cesium Lead Halide Perovskites with High Thermal Stability for Efficient Solar Cells. Adv. Energy Mater. 2016, 6, 1502458. [CrossRef]

2. Xin, Y.C.; Cortecchia, D.; Yin, J.; Bruno, A.; Soci, C. Lead iodide perovskite light-emitting field-effect 332 transistor. Nat. Commun. 2015, 6, 7383. [CrossRef]

3. Lee, Y.; Kwon, J.; Hwang, E.; Ra, C.H.; Yoo, W.J.; Ahn, J.H.; Cho, J.H. High-performance perovskite-graphene 334 hybrid photodetector. Adv. Mater. 2015, 27, 41-46. [CrossRef] [PubMed]

4. Bruzzi, M.; Talamonti, C.; Calisi, N.; Caporali, S.; Vinattieri, A. First proof-of-principle of inorganic perovskites clinical radiotherapy dosimeters. Appl. Phys. Lett. Mat. 2019, 7, 051101. [CrossRef]

5. Ball, J.M.; Petrozza, A. Defects in perovskite-halides and their effects in solar cells. Nat. Energy 2016, 1, 16149. [CrossRef]

6. Polyakov, A.Y.; Smirnov, N.B.; Shchemerov, I.V.; Saranin, D.S.; Le, T.S.; Didenko, S.I.; Kuznetsov, D.V.; Agresti, A.; Pescetelli, S.; Matteocci, F.; et al. Trap states in multication mesoscopic perovskite solar cells: A deep levels transient spectroscopy investigation. Appl. Phys. Lett. 2018, 113, 263501. [CrossRef]

7. Blood, P.; Orton, J.W. The Electrical Characterization of Semiconductors: Majority Carriers and Electron States; Academic Press: Waltham, MA, USA, 1992.

8. Zhang, M.; Zheng, Z.; Fu, Q.; Guo, P.; Zhang, S.; Chen, C.; Chen, H.; Wang, M.; Luo, W.; Tian, Y. Determination of Defect Levels in Melt-Grown All-Inorganic Perovskite $\mathrm{CsPbBr}_{3}$ Crystals by Thermally Stimulated Current Spectra. J. Phys. Chem. C 2018, 122, 10309-10315. [CrossRef]

9. Bruzzi, M.; Gabelloni, F.; Calisi, N.; Caporali, S.; Vinattieri, A. Defective States in Micro-Crystalline CsPbBr3 and Their Role on Photoconductivity. Nanomaterials 2019, 9, 177. [CrossRef]

10. Sherkar, T.S.; Momblona, C.; Gil-Escrig, L.; Avila, J.; Sessolo, M.; Bolink, H.J.; Koster, L.J.A. Recombination in Perovskite Solar Cells: Significance of Grain Boundaries, Interface Traps, and Defect Ions. ACS Energy Lett. 2017, 2, 1214-1222. [CrossRef] 
11. Tress, W.; Marinova, N.; Inganas, O.; Nazeeruddin, M.K.; Zakeeruddin, S.M.; Graetzel, M. Predicting the Open-Circuit Voltage of $\mathrm{CH}_{3} \mathrm{NH}_{3} \mathrm{PbI}_{3}$, Perovskite Solar Cells Using Electroluminescence and Photovoltaic Quantum Effi ciency Spectra: The Role of Radiative and Non-Radiative Recombination. Adv. Energy Mater. 2015, 5, 1400812. [CrossRef]

12. Shao, Y.; ZXiao, Z.; Bi, C.; Yuan, Y.; Huang, J. Origin and elimination of photocurrent hysteresis by fullerene passivation in $\mathrm{CH}_{3} \mathrm{NH}_{3} \mathrm{PbI}_{3}$ planar heterojunction solar cells. Nat. Commun. 2014, 5, 5784. [CrossRef] [PubMed]

13. Akkerman, Q.A.; Genaro Motti, S.; Srimath Kandada, A.R.; Mosconi, E.; D'Innocenzo, V.; Bertoni, G.; Marras, S.; Kamino, B.A.; Miranda, L.; De Angelis, F.; et al. Solution Synthesis Approach to Colloidal Cesium Lead Halide Perovskite Nanoplatelets with Monolayer-Level Thickness Control. J. Am. Chem. Soc. 2016, 138, 1010-1016. [CrossRef] [PubMed]

14. Calisi, N.; Caporali, S.; Milanesi, A.; Innocenti, M.; Salvietti, E.; Bardi, U. Composition-Dependent Degradation of Hybrid and Inorganic Lead Perovskites in Ambient Conditions. Top. Catal. 2018, 61, 1201-1208. [CrossRef]

15. Rakita, Y.; Kedem, N.; Gupta, S.; Sadhanala, A.; Kalchenko, V.; Böhm, M.L.; Kulbak, M.; Friend, R.H.; Cahen, D.; Hodes, G. Low-Temperature Solution-Grown CsPbBr 3 Single Crystals and Their Characterization. Cryst. Growth Des. 2016, 16, 5717-5725. [CrossRef]

16. Gabelloni, F.; Biccari, F.; Falsini, N.; Calisi, N.; Caporali, S.; Vinattieri, A. Long-living nonlinear behavior in $\mathrm{CsPbBr}_{3}$ carrier recombination dynamics. Nanophotonics 2019, 8, 1447-1455. [CrossRef]

17. He, M.; Wang, C.; Li, J.; Wu, J.; Zhang, S.; Kuo, H.C.; Shao, L.; Zhao, S.; Zhang, J.; Kang, F.; et al. $\mathrm{CsPbBr}_{3}-\mathrm{Cs}_{4} \mathrm{PbBr}_{6}$ composite nanocrystals for highly efficient pure green light emission. Nanoscale 2019, 47, 22899-22906. [CrossRef] [PubMed]

18. Sebastian, M.; Peters, J.A.; Stoumpos, C.C.; Im, J.; Kostina, S.S.; Liu, Z.; Kanatzidis, M.G.; Freeman, A.J.; Wessels, B.W. Excitonic emissions and above-bandgap luminescence in the single-crystal perovskite semiconductors $\mathrm{CsPbBr}_{3}$ and $\mathrm{CsPbCl}_{3}$. Phys. Rev. B 2015, 92, 235210. [CrossRef]

19. Dey, A.; Rathod, P.; Kabra, D. Role of Localized States in Photoluminescence Dynamics of High Optical Gain $\mathrm{CsPbBr}_{3}$ Nanocrystals. Adv. Opt. Mater. 2018, 6, 1800109. [CrossRef]

20. Chen, C.; Fu, Q.; Guo, P.; Chen, H.; Wang, M.; Luo, W.; Zheng, Z. Ionic transport characteristics of large-size $\mathrm{CsPbBr} 3$ single crystals. Mater. Res. Express 2019, 6, 115808. [CrossRef]

21. Zhang, B.B.; Wang, F.; Zhang, H.; Xiao, B.; Sun, Q.; Guo, J.; Hafsia, A.B.; Shao, A.; Xu, Y.; Zhou, J. Defect proliferation in $\mathrm{CsPbBr} 3$ crystal induced by ion migration. Appl. Phys. Lett. 2020, 116, 063505. [CrossRef]

22. Sze, S.M.; Ng, K.K. Physics of Semiconductor Devices; John Wiley \& Sons, Inc.: Hoboken, NJ, USA, 2007.

23. Motta, C.; El-Mellouhi, F.; Sanvito, S. Charge carrier mobility in hybrid halide perovskites. Sci. Rep. 2014, 5, 12746. [CrossRef] [PubMed]

24. Savenije, T.J.; Ponseca, C.S., Jr.; Kunneman, L.; Abdellah, M.; Zheng, K.; Tian, Y.; Zhu, Q.; Canton, S.E.; Scheblykin, I.G.; Pullerits, T.; et al. Thermally Activated Exciton Dissociation and Recombination Control the Carrier Dynamics in Organometal Halide Perovskite. J. Phys. Chem. Lett. 2014, 5, 2189-2194. [CrossRef] [PubMed]

25. Duan, H.-S.; Zhou, H.; Chen, Q.; Sun, P.; Luo, S.; Song, T.-B.; Bob, B.; Yang, Y. The identification and characterization of defect states in hybrid organic-inorganic perovskite photovoltaics. Phys. Chem. Chem. Phys. 2015, 17, 112-116. [CrossRef] [PubMed]

26. Baumann, A.; Väth, S.; Rieder, P.; Heiber, M.C.; Tvingstedt, K.; Dyakonov, V. Identification of Trap States in Perovskite Solar Cells. J. Phys. Chem. Lett. 2015, 6, 2350-2354. [CrossRef]

27. Rosenberg, J.W.; Legodi, M.J.; Rakita, Y.; Cahen, D.; Diale, M. Laplace current deep level transient spectroscopy measurements of defect states in methylammonium lead bromide single crystals. J. App. Phys. 2017, 122, 145701. [CrossRef]

28. Kang, J.; Wang, L.-W. High Defect Tolerance in Lead Halide Perovskite CsPbBr 3 . J. Phys. Chem. Lett. 2017, 8, 489-493. [CrossRef]

29. Kovalenko, M.V.; Protesescu, L.; Bodnarchuk, M.I. Properties and potential optoelectronic applications of lead halide perovskite nanocrystals. Science 2017, 358, 745-750. [CrossRef]

30. Whalley, L.D.; Frost, J.M.; Jung, Y.K.; Walsh, A. Perspective: Theory and simulation of hybrid halide perovskites. J. Chem. Phys. 2017, 146, 220901. [CrossRef] 
31. Becker, M.A.; Vaxenburg, R.; Nedelcu, G.; Sercel, P.C.; Shabaev, A.; Mehl, M.J.; Michopoulos, J.G.; Lambrakos, S.G.; Bernstein, N.; Lyons, J.L.; et al. Bright triplet excitons in lead halide perovskites. arXiv 2017, arXiv:1707.03071. [CrossRef]

32. Miyata, K.; Meggiolaro, D.; Tuan Trinh, M.; Joshi, P.P.; Mosconi, E.; Jones, S.C.; De Angelis, F.; Zhu, X.-Y. Large polarons in lead halide perovskites. Mater. Sci. Suppl. Mater. Sci. Adv. 2017, 3, e1701217. [CrossRef]

(C) 2020 by the authors. Licensee MDPI, Basel, Switzerland. This article is an open access article distributed under the terms and conditions of the Creative Commons Attribution (CC BY) license (http://creativecommons.org/licenses/by/4.0/). 